\title{
Monocular SLAM Supported Object Recognition
}

\author{
Sudeep Pillai and John J. Leonard \\ Computer Science and Artificial Intelligence Laboratory \\ Massachusetts Institute of Technology \\ \{spillai, jleonard\}@csail.mit.edu
}

\begin{abstract}
In this work, we develop a monocular SLAMaware object recognition system that is able to achieve considerably stronger recognition performance, as compared to classical object recognition systems that function on a frame-by-frame basis. By incorporating several key ideas including multi-view object proposals and efficient feature encoding methods, our proposed system is able to detect and robustly recognize objects in its environment using a single RGB camera in near-constant time. Through experiments, we illustrate the utility of using such a system to effectively detect and recognize objects, incorporating multiple object viewpoint detections into a unified prediction hypothesis. The performance of the proposed recognition system is evaluated on the UW RGB-D Dataset, showing strong recognition performance and scalable run-time performance compared to current state-of-the-art recognition systems.
\end{abstract}

\section{INTRODUCTION}

Object recognition is a vital component in a robot's repertoire of skills. Traditional object recognition methods have focused on improving recognition performance (Precision-Recall, or mean Average-Precision) on specific datasets $[17,29]$. While these datasets provide sufficient variability in object categories and instances, the training data mostly consists of images of arbitrarily picked scenes and/or objects. Robots, on the other hand, perceive their environment as a continuous image stream, observing the same object several times, and from multiple viewpoints, as it constantly moves around in its immediate environment. As a result, object detection and recognition can be further bolstered if the robot were capable of simultaneously localizing itself and mapping (SLAM) its immediate environment - by integrating object detection evidences across multiple views.

We refer to a "SLAM-aware" system as - one that has access to the map of its observable surroundings as it builds it incrementally and the location of its camera at any point in time. This is in contrast to classical recognition systems that are "SLAM-oblivious" - those that detect and recognize objects on a frame-by-frame basis without being cognizant of the map of its environment, the location of its camera, or that objects may be situated within these maps. In this paper, we develop the ability for a SLAM-aware system to robustly recognize objects in its environment, using an RGB camera as its only sensory input (Figure 1).

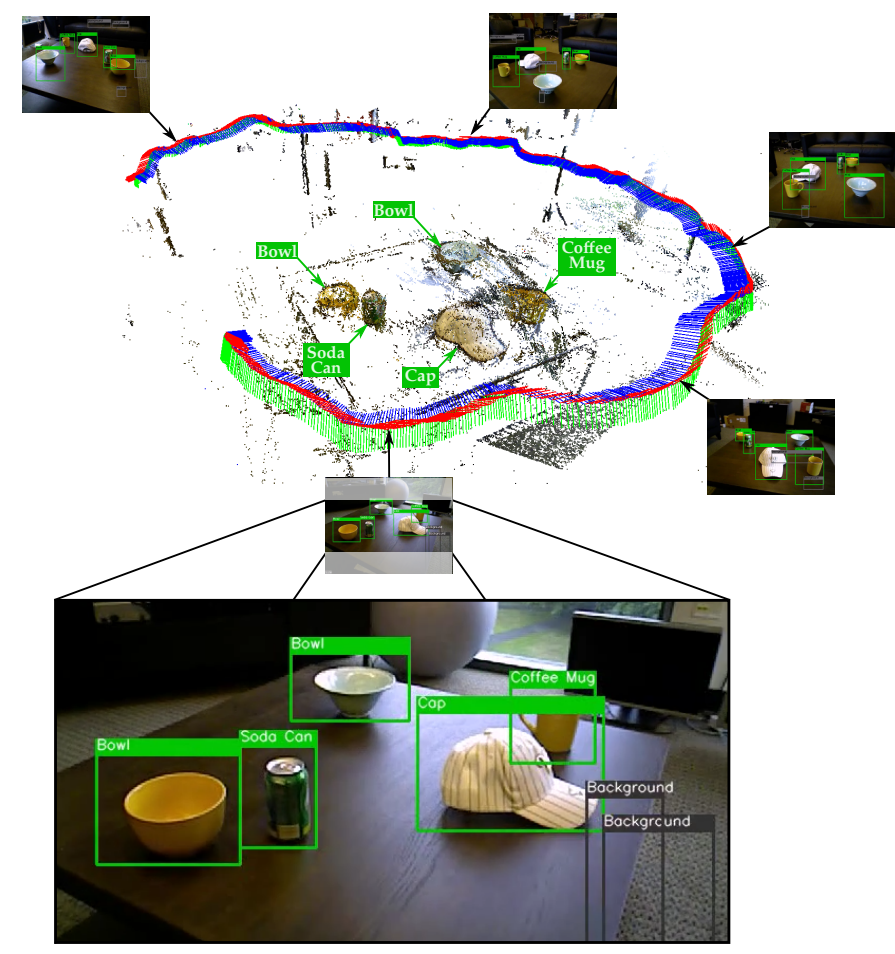

Fig. 1: The proposed SLAM-aware object recognition system is able to robustly localize and recognize several objects in the scene, aggregating detection evidence across multiple views. Annotations in white are provided for clarity and are actual predictions proposed by our system.

We make the following contributions towards this end: Using state-of-the-art semi-dense map reconstruction techniques in monocular visual SLAM as pre-processed input, we introduce the capability to propose multiview consistent object candidates, as the camera observes instances of objects across several disparate viewpoints. Leveraging this object proposal method, we incorporate some of the recent advancements in bag-of-visual-wordsbased (BoVW) object classification [1, 15, 22] and efficient box-encoding methods [34] to enable strong recognition performance. The integration of this system with a monocular visual-SLAM (vSLAM) back-end also enables us to take advantage of both the reconstructed map and camera location to significantly bolster recognition performance. Additionally, our system design allows the run-time performance to be scalable to a larger number of object categories, with near-constant run-time for most 


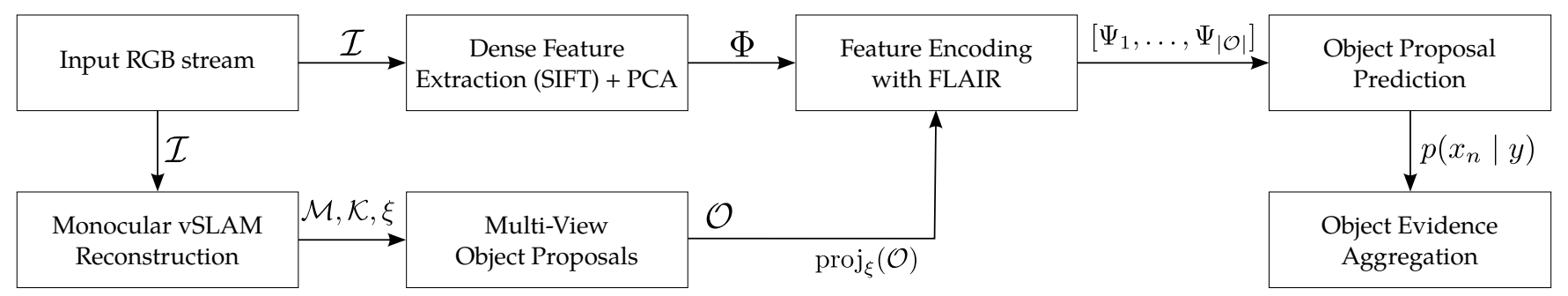

Fig. 2: Outline of the SLAM-aware object recognition pipeline. Given an input RGB image stream $\mathcal{I}$, we first reconstruct the scene in a semi-dense fashion using an existing monocular visual-SLAM implementation (ORB-SLAM) with a semi-dense depth estimator, and subsequently extract relevant map $\mathcal{M}$, keyframe $\mathcal{K}$ and pose information $\xi$. We perform multi-scale density-based segmentation on the reconstructed scene to obtain object proposals $\mathcal{O}$ that are consistent across multiple views. On each of the images in the input RGB image stream $\mathcal{I}$, we compute Dense-SIFT $\left(\mathbb{R}^{128}\right)+R G B\left(\mathbb{R}^{3}\right)$ and reduce it to $\Phi \in \mathbb{R}^{80}$ via PCA. The features $\Phi$ are then used to efficiently encode each of the projected object proposals $\mathcal{O}$ (bounding boxes of proposals projected on to each of the images with known poses $\xi$ ) using VLAD with FLAIR, to obtain $\Psi$. The resulting feature vector $\Psi$ is used to train and predict likelihood of target label/category $p\left(x_{i} \mid y\right)$ of the object contained in each of the object proposals. The likelihoods for each object $o \in \mathcal{O}$ are aggregated across each of the viewpoints $\xi$ to obtain robust object category prediction.

practical object recognition tasks.

We present several experimental results validating the improved object proposition and recognition performance of our proposed system: (i) The system is compared against the current state-of-the-art [24, 25] on the UW-RGBD Scene [23, 25] Dataset. We compare the improved recognition performance of being SLAMaware, to being SLAM-oblivious (ii) The multi-view object proposal method introduced is shown to outperform single-view object proposal strategies such as BING [9] on the UW-RGBD dataset, that provide object candidates solely on a single-view. (iii) The run-time performance of our system is analysed, with specific discussion on the scalability of our approach, compared to existing stateof-the-art methods [24, 25].

\section{Related Work}

We discuss some of the recent developments in object proposals, recognition, and semi-dense monocular visual SLAM literature that has sparked the ideas explained in this paper.

Sliding window techniques and DPM In traditional state-of-the-art object detection, HOG [13] and deformable-part-based-models (DPM) proposed by Felzenszwalb et al. [18] have become the norm due to their success in recognition performance. These methods explicitly model the shape of each object and its parts via oriented-edge templates, across several scales. Despite its reduced dimensionality, the template model is scanned over the entire image in a sliding-window fashion across multiple scales for each object type that needs to be identified. This is a highly limiting factor in scalability, as the run-time performance of the system is directly dependent on the number of categories identifiable. While techniques have been proposed to scale such schemes to larger object categories [14], they incur a drop in recognition performance to trade-off for speed.

Dense sampling and feature encoding methods Recently, many of the state-of-the-art techniques [26, 34] for generic object classification have resorted to dense feature extraction. Features are densely sampled on an image grid [5], described, encoded and aggregated over the image or a region to provide a rich description of the object contained in it. The aggregated feature encodings lie as feature vectors in high-dimensional space, on which linear or kernel-based classification methods perform remarkably well. Among the most popular encoding schemes include Bag-of-Visual-Words (BoVW) $[12,31]$, and more recently Super-Vectors [35], VLAD [22], and Fisher Vectors [28]. In the case of BoVW, a histogram of occurrences of codes are built using a vocabulary of finite size $V \in \mathbb{R}^{K \times D}$. VLAD and Fisher Vectors, in contrast, aggregate residuals using the vocabulary to estimate the first and second order moment statistics in an attempt to reduce the loss of information introduced in the vector-quantization (VQ) step in BoVW. Both VLAD and Fisher Vectors have been shown to outperform traditional BoVW approaches [8, 22, 28], and are used as a drop-in replacement to BoVW; we do the same utilizing VLAD as it provides a good trade-off between descriptiveness and computation time.

Object Proposals Recently, many of the state-of-theart techniques in large-scale object recognition systems have argued the need for a category-independent object proposal method that provides candidate regions in images that may likely contain objects. Variants of these include Constrained-Parametric Min-cuts (CPMC) [6], Selective Search [33], Edge Boxes [36], Binarized Normed Gradients (BING) [9]. The object candidates proposed are category-independent, and achieve detection rates (DR) of $95-99 \%$ at 0.7 intersection-over-union ( $\left.\mathrm{IoU}^{1}\right)$ threshold, by generating about 1000-5000 candidate proposal windows [21, 36]. This dramatically reduces the search space for existing sliding-window approaches that scan templates over the entire image, and across multiple scales; however, it still bodes a challenge to accurately

\footnotetext{
${ }^{1}$ Intersection-over-Union (IoU) is a common technique to evaluate the quality of candidate object proposals with respect to ground truth. The intersection area of the ground truth bounding box and that of the candidate is divided by the union of their areas.
} 
classify irrelevant proposal windows as background. For a thorough evaluation of the state-of-the-art object proposal methods, and their performance, we refer the reader to Hosang et al. [21].

Scalable Encoding with Object Proposals As previously addressed, sliding-window techniques inherently deal with the scalability issue, despite recent schemes to speed-up such an approach. BoVW, on the contrary, handle this scalability issue rather nicely since the histograms do not particularly encode spatial relations as strongly. This however, makes BoVW approaches lack the ability to localize objects in an image. The advent of category-independent object proposal methods have subsequently opened the door to bag-of-words-driven architectures, where object proposal windows can now be described via existing feature encoding methods. Most recently, van de Sande et al. [34] employ a novel box-encoding technique using integral histograms to describe object proposal windows with a run-time independent of the window size of object proposals supplied. They report results with an $18 x$ speedup over brute-force BoVW encoding (for 30,000 object proposals), enabling a new state-of-the-art on the challenging 2010 PASCAL VOC detection task. Additionally their proposed system ranks number one in the official ImageNet 2013 detection challenge, making it a promising solution to consider for robotics applications.

Multi-view Object Detection While classical object detection methods focus on single-view-based recognition performance, some of these methods have been extended to the multi-view case [11,32], by aggregating object evidence across disparate views. Lai et al. [24] proposed a multi-view-based approach for detecting and labeling objects in a 3D environment reconstructed using an RGB-D sensor. They utilize the popular HOG-based sliding-window detectors trained from object views in the RGB-D dataset $[23,25]$ to assign class probabilities to pixels in each of the frames of the RGB-D stream. Given co-registered image and depth, these probabilities are assigned to voxels in a discretized reconstructed 3D scene, and further smoothed using a Markov Random Field (MRF). Bao et al. [2, 3] proposed one of the first approaches to jointly estimate camera parameters, scene points and object labels using both geometric and semantic attributes in the scene. In their work, the authors demonstrate the improved object recognition performance, and robustness by estimating the object semantics and SfM jointly. However, the run-time of 20 minutes per image-pair, and the limited object categories identifiable makes the approach impractical for on-line robot operation. Other works $[4,7,10,20,30]$ have also investigated object-based SLAM, SLAM-aware, and $3 \mathrm{D}$ object recognition architectures, however they have a few of glaring concerns: either (i) the system cannot scale beyond a finite set of object instances (generally limited to less than 10), or (ii) they require RGB-D input to support both detection and pose estimation, or (iii) they require rich object information such as $3 \mathrm{D}$ models in its database to match against object instances in a bruteforce manner.

\section{Monocular SLAM SUPPORTEd Object Recognition}

This section introduces the algorithmic components of our method. We refer the reader to Figure 2 that illustrates the steps involved, and provide a brief overview of our system.

\section{A. Multi-view Object Proposals}

Most object proposal strategies use either superpixelbased or edge-based representations to identify candidate proposal windows in a single image that may likely contain objects. Contrary to classical per-frame object proposal methodologies, robots observe the same instances of objects in its environment several times and from disparate viewpoints. It is natural to think of object proposals from a spatio-temporal or reconstructed $3 \mathrm{D}$ context, and a key realization is the added robustness that the temporal component provides in rejecting spatially inconsistent edge observations or candidate proposal regions. Recently, Engel et al. [16] proposed a scaledrift aware monocular visual SLAM solution called LSDSLAM, where the scenes are reconstructed in a semidense fashion, by fusing spatio-temporally consistent scene edges. Despite being scale-ambivalent, the multiview reconstructions can be especially advantageous in teasing apart objects in the near-field versus those in the far-field regions, and thus subsequently be useful in identifying candidate object windows for a particular view. We build on top of an existing monocular SLAM solution (ORB-SLAM [27]) and augment a semidense depth filtering component derived from [19]. The resulting reconstruction qualitatively is similar to that produced by LSD-SLAM [16], and is used for subsequent object proposal generation. We avoided the use of LSD-SLAM as it occasionally failed over tracking widebaseline motions inherent in the benchmark dataset we used.

In order to retrieve object candidates that are spatiotemporally consistent, we first perform a density-based partitioning on the scale-ambiguous reconstruction using both spatial and edge color information. This is done repeatedly for 4 different density threshold values (each varied by a factor of 2), producing an over-segmentation of points in the reconstructed scene that are used as seeds for multi-view object candidate proposal. The spatial density segmentations eliminate any spurious points or edges in the scene, and the resulting point cloud is sufficient for object proposals. These object over-segmentation seeds are subsequently projected onto each of the camera views, and serve as seeds to for further occlusion handling, refinement and candidate 

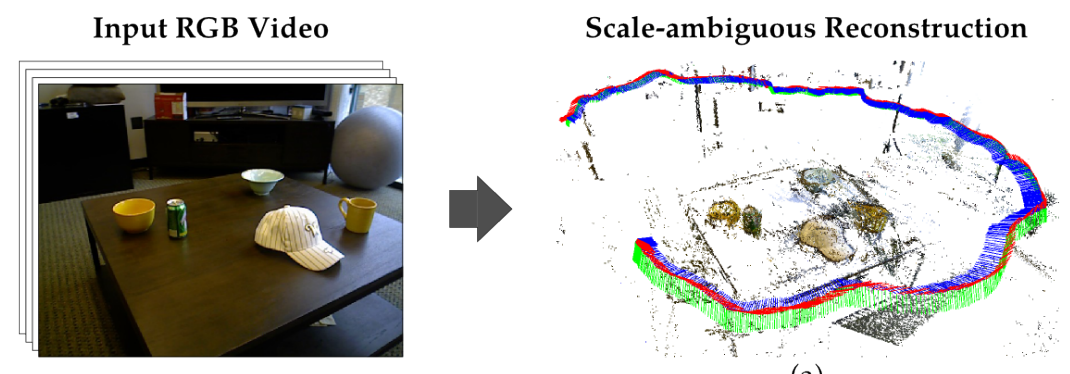

(a)

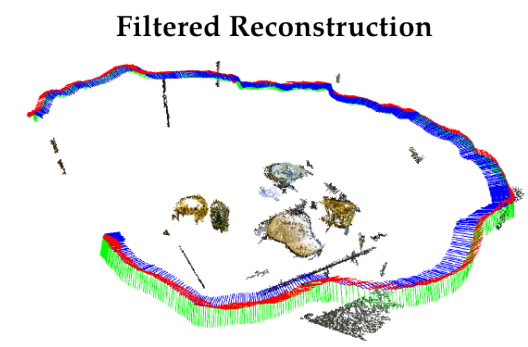

(b)

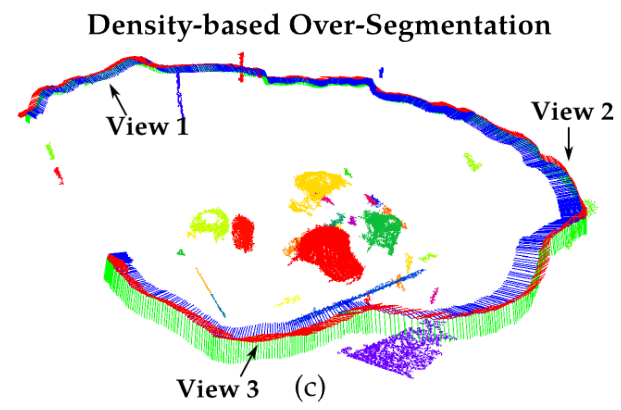

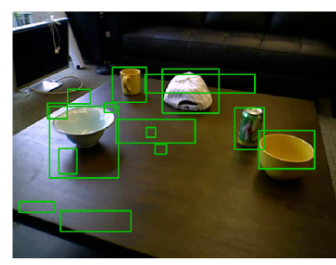

(c)

Multi-view object proposals

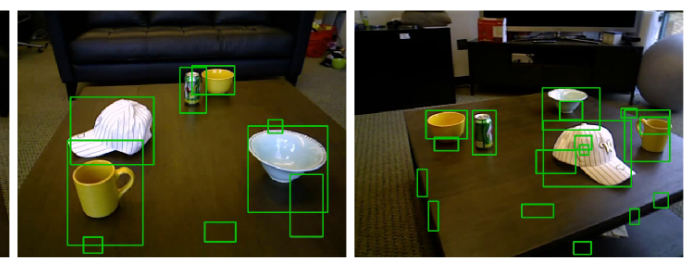

(d)
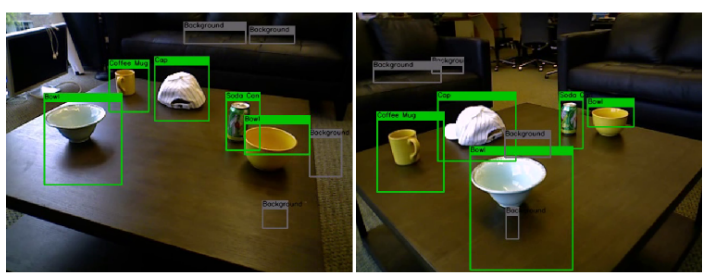

SLAM-aware object detection
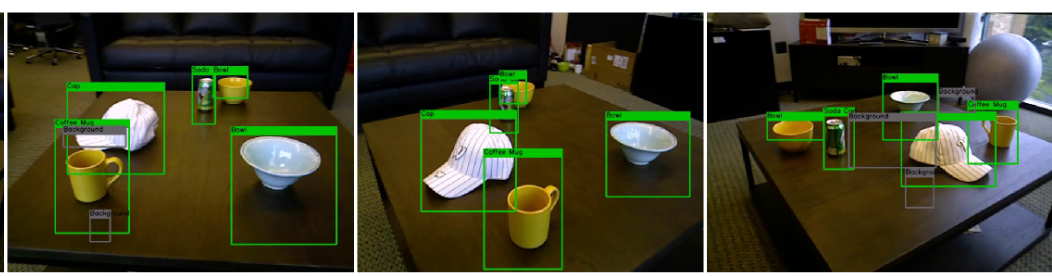

(e)

Fig. 3: An illustration of the multi-view object proposal method and subsequent SLAM-aware object recognition. Given an input RGB image stream, a scale-ambiguous semi-dense map is reconstructed (a) via the ORB-SLAM-based [27] semi-dense mapping solution. The reconstruction retains edges that are consistent across multiple views, and is employed in proposing objects directly from the reconstructed space. The resulting reconstruction is (b) filtered and (c) partitioned into several segments using a multi-scale density-based clustering approach that teases apart objects (while filtering out low-density regions) via the semi-dense edge-map reconstruction. Each of the clustered regions are then (d) projected on to each of individual frames in the original RGB image stream, and a bounded candidate region is proposed for subsequent feature description, encoding and classification. (e) The probabilities for each of the proposals per-frame are aggregated across multiple views to infer the most likely object label.

object proposal generation. We cull out (i) small candidates whose window size is less than $20 \times 20 \mathrm{px}$, (ii) occluding candidates by estimating their median depth from the reconstruction, to avoid mis-identification and (iii) overlapping candidates with an IoU threshold of 0.5, to avoid redundant proposals. The filtered set of windows are subsequently considered as candidates for the classification process downstream. Figure 3 illustrates the different steps described in this section.

\section{B. State-of-the-art Bag-of-Visual-Words with Object Proposals}

Given the object proposals computed using the reconstructed scale-ambiguous map, we now direct our attention to describing these proposal regions.

Dense BoVW with VLAD Given an input image and candidate object proposals, we first densely sample the image, describing each of the samples with SIFT + RGB color values, $\Phi_{S I F T+R G B} \in \mathbb{R}^{131}$ i.e. Dense SIFT (128-D) + RGB(3-D). Features are extracted with a step size of 4 pixels, and at 4 different pyramid scales with a pyramid scale factor of $\sqrt{2}$. The resulting description is then reduced to a 80-dimensional vector via PCA, called PCASIFT $\Phi \in \mathbb{R}^{80}$. A vocabulary $V \in \mathbb{R}^{K \times 80}$ of size $K=64$ is created via $k$-means, using the descriptions extracted from a shuffled subset of the training data, as done in classical bag-of-visual-words approaches. In classical BoVW, this vocabulary can be used to encode each of the original SIFT+RGB descriptions in an image into a histogram of occurrences of codewords, which in turn provides a compact description of the original image. Recently, however, more descriptive encodings such as VLAD [22] and Fisher Vectors [28] have been shown to outperform classical BoVW approaches [8, 22, 28]. Consequently, we chose to describe the features using VLAD as it provides equally as strong performance with slightly reduced computation time as compared to Fisher Vectors.

For each of the bounding boxes, the un-normalized VLAD $\Psi \in \mathbb{R}^{K D}$ description is computed by aggregating the residuals of each of the descriptions $\Phi$ (enclosed 


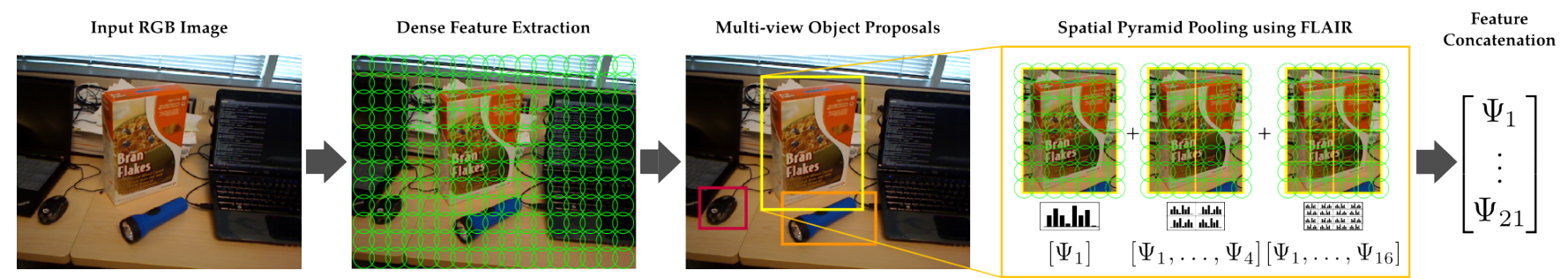

Fig. 4: Various steps involved in the feature extraction procedure. Features that are densely sampled from the image are subsequently used to describe the multi-view object proposals using FLAIR. Each proposal is described with multiple ([1x1], [2x2], [4x4]) spatial levels/bins via quick table lookups in the integral VLAD histograms (through FLAIR). The resulting histogram $\Psi$ (after concatenation) is used to describe the object contained in the bounding box. Figure is best viewed in electronic form.

within the bounding box) from their vector-quantized centers in the vocabulary, thereby determining its first order moment (Eq. 1).

$$
v_{k}=\sum_{x_{i}: N N\left(x_{i}\right)=\mu_{k}} x_{i}-\mu_{k}
$$

The description is then normalized using signed-squarerooting (SSR) or commonly known as power normalization (Eq. 2) with $\alpha=0.5$, followed by L2 normalization, for improved recognition performance as noted in [1].

$$
f(z)=\operatorname{sign}(z)|z|^{\alpha} \quad \text { where } \quad 0 \leq \alpha \leq 1
$$

Additional descriptions for each bounding region are constructed for 3 different spatial bin levels or subdivi-

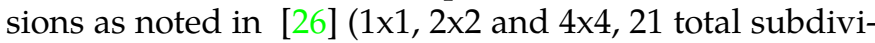
sions $S$ ), and stacked together to obtain the feature vector $\Psi=\left[\ldots \mathbf{v}_{s} \ldots\right] \in \mathbb{R}^{K D S}$ that appropriately describes the specific object contained within the candidate object proposal/bounding box.

Efficient Feature Encoding with FLAIR While it may be practical to describe a few object proposals in the scene with these encoding methods, it can be highly impractical to do so as the number of object proposals grows. To this end, van de Sande et al. [34] introduced FLAIR - an encoding mechanism that utilizes summedarea tables of histograms to enable fast descriptions for arbitrarily many boxes in the image. By constructing integral histograms for each code in the codebook, the histograms or descriptions for an arbitrary number of boxes $B$ can be computed independent of their area. As shown in [34], these descriptions can also be extended to the VLAD encoding technique. Additionally, FLAIR affords performing spatial pyramid binning rather naturally, with only requiring a few additional table look-ups, while being independent of the area of $B$. We refer the reader to Figure 4 for an illustration of the steps involved in describing these candidate object proposals.

Multi-class histogram classification Given training examples, $\left(x_{1}, y_{1}\right), \ldots,\left(x_{n}, y_{n}\right)$ where $x_{i} \in \mathbb{R}^{K D S}$ are the VLAD descriptions and $y_{i} \in\{1, \ldots, \mathcal{C}\}$ are the ground truth target labels, we train a linear classifier using
Stochastic Gradient Descent (SGD), given by:

$$
E(w)=\frac{1}{n} \sum_{i=1}^{n} L\left(y_{i}, f\left(x_{i}\right)\right)+\alpha R(w)
$$

where $L\left(y_{i}, f\left(x_{i}\right)\right)=\log \left(1+\exp \left(-y_{i} \mathbf{w}^{T} \mathbf{x}_{i}\right)\right)$ is the logistic loss function, $R(w)=\frac{1}{2} \sum_{i=1}^{n} \mathbf{w}^{T} \mathbf{w}$ is the L2regularization term that penalizes model complexity, and $\alpha>0$ is a non-negative hyperparameter that adjusts the $\mathrm{L} 2$ regularization. A one-versus-all strategy is taken to extend the classifiers to multi-class categorization. For hard-negative mining, we follow [34] closely, bootstrapping additional examples from wrongly classified negatives for 2 hard-negative mining epochs.

\section{Multi-view Object Recognition}

We start with the ORB-SLAM-based semi-dense mapping solution, that feeds a continuous image stream, in order to recover a scale-ambiguous map $\mathcal{M}$, keyframes $\mathcal{K}$, and poses $\xi$ corresponding to each of the frames in the input image stream. The resulting scaleambiguous reconstruction provides a strong indicator of object presence in the environment, that we use to over-segment into several object seeds $o \in\{1, \ldots, \mathcal{O}\}$. These object seeds are projected back in to each of the individual frames using the known projection matrix, derived from its corresponding viewpoint $\xi_{i}$. The median depth estimates of each of the seeds are estimated in order to appropriately project non-occluding object proposals back in to corresponding viewpoint, using a depth buffer. Using these as candidate object proposals, we evaluate our detector on each of the $\mathcal{O}$ object clusters, per image, providing probability estimates of belonging to one of the $\mathcal{C}$ object classes or categories. Thus, the maximum-likelihood estimate of the object $o \in \mathcal{O}$ can be formalized as maximizing the data-likelihood term for all observable viewpoints (assuming uniform prior across the $\mathcal{C}$ classes):

$$
\hat{y}^{M L E}=\underset{y \in\{1, \ldots,|\mathcal{C}|\}}{\operatorname{argmax}} p\left(\mathcal{D}_{o} \mid y\right) \quad \forall o \in \mathcal{O}
$$

where $y \in\{1, \ldots,|\mathcal{C}|\}$ are the class labels, $\mathcal{D}_{o}=$ $\left\{x_{1}, \ldots, x_{N}\right\}_{o}$ is the data observed of the object cluster $o \in \mathcal{O}$ across $N$ observable viewpoints. In our case, $\mathcal{D}_{o}$ 

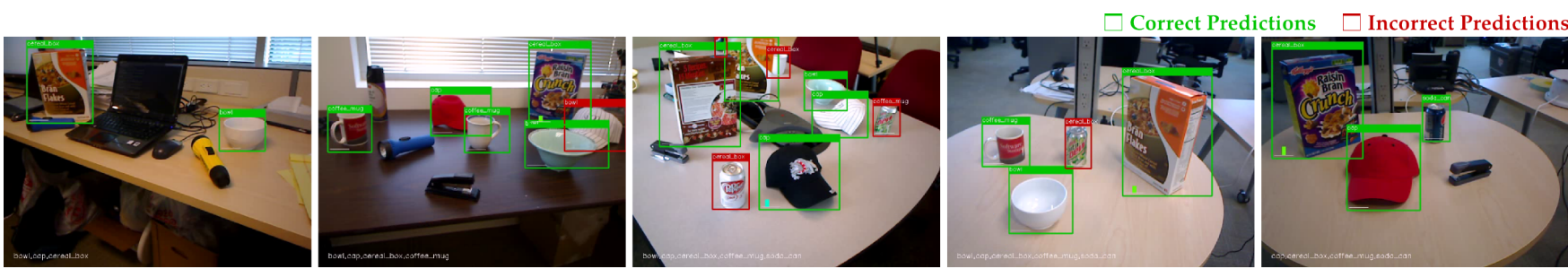

Fig. 5: Illustration of per-frame detection results provided by our object recognition system that is intentionally SLAM-oblivious (for comparison purposes only). Object recognition evidence is not aggregated across all frames, and detections are performed on a frame-by-frame basis. Only detections having corresponding ground truth labels are shown. Figure is best viewed in electronic form.

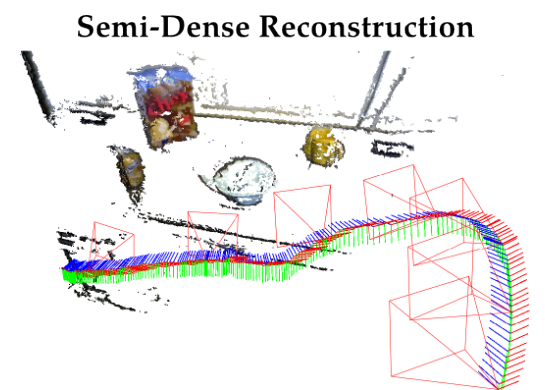

Multi-View Object Proposals

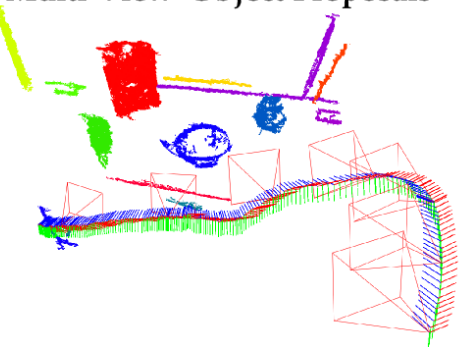

Frame-based Object Recognition

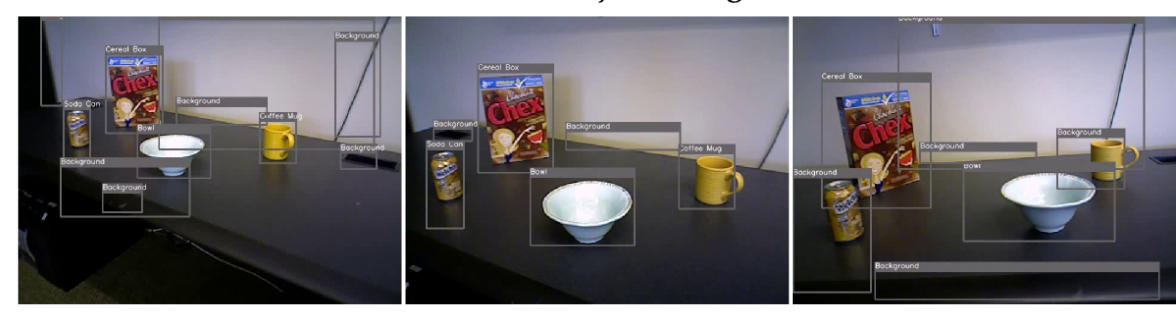

SLAM-aware Object Recognition

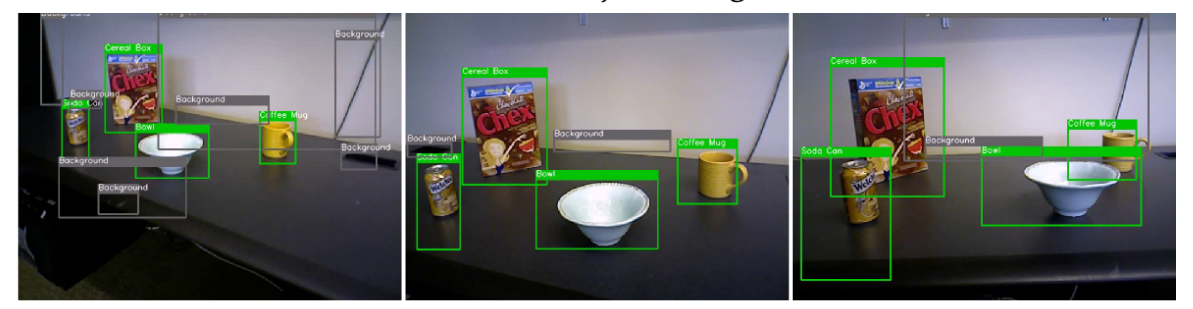

Fig. 6: Illustration of the recognition capabilities of our proposed SLAM-aware object recognition system. Each of the object categories are detected every frame, and their evidence is aggregated across the entire sequence through the set of object hypothesis. In frame-based object recognition, predictions are made on an individual image basis (shown in gray). In SLAM-aware recognition, the predictions are aggregated across all frames in the image sequence to provide robust recognition performance. The green boxes indicate correctly classified object labels, and the gray boxes indicate background object labels. Figure is best viewed in electronic form.

refers to the bounding box of the $o^{\text {th }}$ cluster, projected onto each of the $N$ observable viewpoints. Assuming the individual features in $\mathcal{D}_{o}$ are conditionally independent given the class label $y$, the maximum-likelihood estimate (MLE) factorizes to:

$$
\begin{aligned}
\hat{y}^{M L E} & =\underset{y \in\{1, \ldots,|\mathcal{C}|\}}{\operatorname{argmax}} \prod_{n=1}^{N} p\left(x_{n} \mid y\right) \\
& =\underset{y \in\{1, \ldots,|\mathcal{C}|\}}{\operatorname{argmax}} \sum_{n=1}^{N} \log p\left(x_{n} \mid y\right)
\end{aligned}
$$

Thus the MLE of an object cluster $o$ belonging to one of the $\mathcal{C}$ classes, is the class that corresponds to having the highest of the sum of the log-likelihoods of their individual class probabilities estimated for each of the $N$ observable viewpoints.

\section{EXPERIMENTS}

In this section, we evaluate the proposed SLAMaware object recognition method. In our experiments, we extensively evaluate our SLAM-aware recognition system on the popular UW RGB-D Dataset (v2)[23, 25].
We compare against the current state-of-the-art solution proposed by Lai et al. [24], that utilize full map and camera location information for improved recognition performance. The UW RGB-D dataset contains a total 51 object categories, however, in order to maintain a fair comparison, we consider the same set of 5 objects as noted in [24]. In experiment 3, we propose scalable recognition solutions, increasing the number of objects considered to all 51 object categories in the UW RGB-D Dataset.

Experiment 1: SLAM-Aware Object Recognition Performance Evaluation We train and evaluate our system on the UW RGB-D Scene Dataset [23, 25], providing mean-Average Precision (mAP) estimates (see Table I) for the object recognition task and compare against existing methods [24]. We split our experiments into two categories:

(i) Single-View recognition performance: First, we evaluate the recognition performance of our proposed system on each of the scenes in the UW-RGB-D Scene Dataset on a per-frame basis, detecting and classifying objects 


\begin{tabular}{|c|c|c|c|c|c|c|c|c|c|}
\hline \multirow{2}{*}{ Method } & \multirow{2}{*}{ View(s) } & \multirow{2}{*}{ Input } & \multicolumn{7}{|c|}{ Precision/Recall } \\
\hline & & & Bowl & Cap & Cereal Box & Coffee Mug & Soda Can & Background & Overall \\
\hline DetOnly & Single & RGB & $46.9 / 90.7$ & $54.1 / 90.5$ & $76.1 / 90.7$ & $42.7 / 74.1$ & $51.6 / 87.4$ & 98.8 & 61.7 \\
\hline Det3DMRF [24] & Multiple & RGB-D & $91.5 / 85.1$ & $90.5 / 91.4$ & $93.6 / 94.9$ & $90.0 / 75.1$ & $81.5 / 87.4$ & $99.0 / 99.1$ & $91.0 / 88.8$ \\
\hline $\mathrm{HMP} 2 \mathrm{D}+3 \mathrm{D}$ [25] & Multiple & RGB-D & $97.0 / 89.1$ & $82.7 / 99.0$ & $96.2 / 99.3$ & $81.0 / 92.6$ & $97.7 / 98.0$ & $95.8 / 95.0$ & $90.9 / 95.6$ \\
\hline Ours & Single & RGB & $88.6 / 71.6$ & $85.2 / 62.0$ & $83.8 / 75.4$ & $70.8 / 50.8$ & $78.3 / 42.0$ & 95.0 & $81.5 / 59.4$ \\
\hline Ours & Multiple & RGB & $88.7 / 70.2$ & $99.4 / 72.0$ & $95.6 / 84.3$ & $80.1 / 64.1$ & $89.1 / 75.6$ & $96.6 / 96.8$ & $89.8 / 72.0$ \\
\hline
\end{tabular}

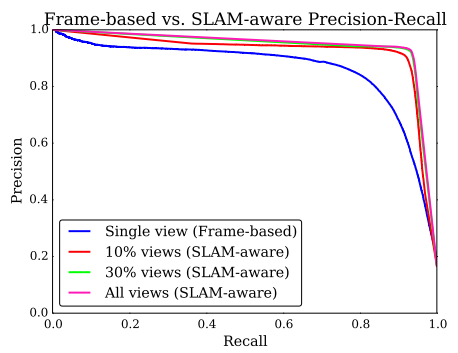

TABLE I \& Fig. 7: Left: Object classification results using the UW RGB-D Scene Dataset [23, 25], providing mean-Average Precision (mAP) estimates for both Single-View, and Multi-View object recognition approaches. We compare against existing methods([24, 25]) that use RGB-D information instead of relying only on RGB images, in our case. Recognition for the single-view approach is done on a per-frame basis, where prediction performance is averaged across all frames across all scenes. For the multi-view approach, recognition is done on a per-scene basis, where prediction performance is averaged across all scenes. Right: Performance comparison via precision-recall for the Frame-based vs. SLAMaware object recognition. As expected, the performance of our proposed SLAM-aware solution increases with more recognition evidence is aggregated across multiple viewpoints.

that occur every 5 frames in each scene (as done in [24]). Each object category is trained from images in the $\mathrm{Ob}$ ject Dataset, that includes several viewpoints of object instances with their corresponding mask, and category information. Using training parameters identical to the previous experiment, we achieve a performance of 81.5 $\mathrm{mAP}$ as compared to the detector performance of 61.7 mAP reported in [24]. Recognition is done on a perimage basis, and averaged across all test images for reporting. Figure 5 shows the recognition results of our system on a per-frame basis. We ignore regions labeled as background in the figure for clarity and only report the correct and incorrect predictions in green and red respectively.

(ii) Multi-View recognition performance: In this section, we investigate the performance of a SLAM-aware object recognition system. We compare this to a SLAMoblivious object detector described previously, and evaluate using ground truth provided. Using the poses $\xi$ and reconstructed map $\mathcal{M}$, multi-view object candidates are proposed and projected onto each of the images for each scene sequence. Using the candidates provided as input to the recognition system, the system predicts the likelihood and corresponding category of an object (including background) contained in a candidate bounding box. For each of the objects $o \in \mathcal{O}$ proposed, the summed log-likelihood is computed (as in Eqn. 4) to estimate the most likely object category over all the images for a particular scene sequence. We achieve $89.8 \mathrm{mAP}$ recognition performance on the 5 objects in each of the scenes in [25] that was successfully reconstructed by the ORB-SLAMbased semi-dense mapping system. Figures 1, 3, 6 and 9 illustrate the capabilities of the proposed system in providing robust recognition performance by taking advantage of the monocular visual SLAM-backend. Figure 7 illustrates the average precision-recall performance on the UW RGB-D dataset, comparing the classical framebased and our SLAM-aware approach. As expected, with additional object viewpoints, our proposed SLAM-aware solution predicts with improved precision and recall. In comparison to that of HMP2D+3D [25], they achieve only slightly higher overall recognition performance of 90.9 $\mathrm{mAP}$, as their recognition pipeline takes advantage of the RGB and depth input to improve overall scene reconstruction. We do note that while we perform comparably with HMP2D+3D [25], our BoVW+FLAIR architecture allows our system to scale to a large number of object categories with near-constant run-time. We investigate the run-time performance and scalability concerns further in Experiment 3.

Experiment 2: Multi-View Objectness In this experiment, we investigate the effectiveness of our multiview object proposal method in identifying categoryindependent objects in a continuous video stream. We compare the recall of our object proposal method with the recently introduced BING [9] object proposal technique, whose performance in detection rate (DR) and run-time claim to be promising. We compare against the BING method, varying the number of proposed object candidates by picking proposals in descending order of their objectness score. Figure 8 compares the overall performance of our multi-view object proposal method that achieves better recall rates, for a particular IoU threshold with considerably fewer object proposals. The results provided are evaluated on all the scenes provided in the UW-RGB-D dataset (v2) [25].

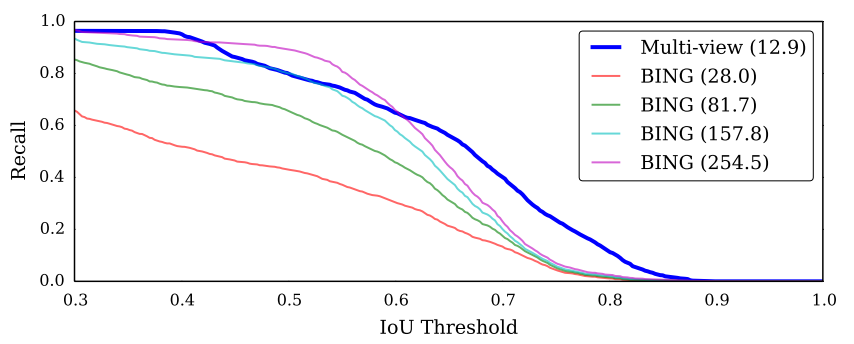

Fig. 8: Varying number of proposals: We experiment with varied number of bounding boxes for the BING object proposal method, and compare against our multi-view object proposal method that uses considerably fewer number of bounding boxes to get similar or better recall rates. The numbers next to the label indicate the average number of windows proposed in the image. 
Experiment 3: Scalable recognition and run-time evaluation In this section, we investigate the run-time performance of computing VLAD with integral histograms (FLAIR) for our system and compare against previously proposed approaches [24, 34]. We measure the average speed for feature-extraction (Dense-SIFT) and feature-encoding (VLAD) as they take up over 95\% of the overall compute time. All experiments were conducted with a single-thread on an Intel Core-i7-3920XM $(2.9 \mathrm{GHz})$.

\begin{tabular}{|c|c|c|c|}
\hline Method & $|\mathcal{C}|$ & Run-time (s) & mAP/Recall \\
\hline DetOnly [24] & 5 & $\approx 1.8 \mathrm{~s}$ & $61.7 / 87.9$ \\
\hline DetOnly [24] & 51 & $\geq 5^{\dagger} \mathrm{s}$ & - \\
\hline HMP2D+3D [25] & 9 & $\approx 4 \mathrm{~s}$ & $92.8 / 95.3$ \\
\hline Ours & 5 & $1.6 \mathrm{~s}$ & $81.5 / 59.4$ \\
\hline Ours & 10 & $1.6 \mathrm{~s}$ & $86.1 / 58.4$ \\
\hline Ours & 51 & $1.7 \mathrm{~s}$ & $75.7 / 60.9$ \\
\hline
\end{tabular}

TABLE II: Analysis of run-time performance of our system (for framebased detection) compared to that of [24] and [25]. We achieve comparable performance, and show scalable recognition performance with a near-constant run-time cost (with increasing number of identifiable object categories $|\mathcal{C}|=51$ ). Existing sliding-window approaches become impractical ( $\geq 4$ s run-time) in cases where $|\mathcal{C}| \approx 51$.

van de Sande et al. [34] reports that the overall feature extraction and encoding takes 5.15s (VQ 0.55s, FLAIR construction 0.6s, VLAD+FLAIR 4.0s) per image, with the following parameters (2px step size, 3 Pyr. Scales, [1x1], [4x4] spatial pyramid bins). With significantly fewer candidate proposals, and careful implementation, our system is able to achieve the same (with $4 p x$ step size) in approximately 1.6s. With reference to [24], where the run-time performance of the sliding-window approach is directly proportional to the number of object categories detectable, the authors report an overall runtime of $1.8 \mathrm{~s}$ for 5 object categories. However, scaling up their detection to larger number of objects would imply costly runtimes, making it highly impractical for realtime purposes. The run-time of our approach (based on [34]), on the other hand, is scalable to a larger number of object categories, making it a strong contender for real-time recognition systems. We summarize the runtimes of our approach compared to that of [24] and [25] in Table II.

Discussion and Future Work While there are benefits to running a monocular visual SLAM-backend for recognition purposes, the inter-dependence of the recognition system on this backend makes it vulnerable to the same robustness concerns that pertain to monocular visual SLAM. In our experiments, we noticed inadequacies in the semi-dense vSLAM implementation that failed to reconstruct the scene on few occasions. To further emphasize recognition scalability, we are actively collecting a larger scaled dataset (in increased map area, and number of objects) to show the extent of capabilities of the proposed system. Furthermore, we realize the

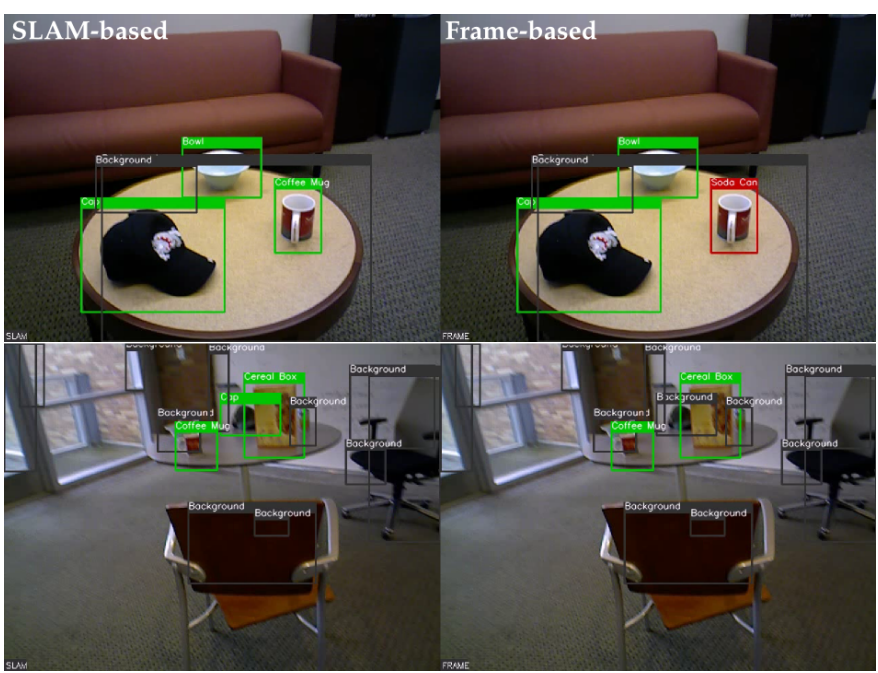

Fig. 9: More illustrations of the superior performance of the SLAMaware object recognition in scenarios of ambiguity and occlusions. The coffee mug is misidentified as a soda can, and the cap in the bottom row is occluded by the cereal box.

importance of real-time capabilities of such recognition systems, and intend to generalize the architecture to a streaming approach in the near future. We also hope to release the source code for our proposed method, allowing scalable and customizable training with fast run-time performance during live operation.

\section{Conclusion}

In this work, we develop a SLAM-aware objectrecognition system, that is able to provide robust and scalable recognition performance as compared to classical SLAM-oblivious recognition methods. We leverage some of the recent advancements in semi-dense monocular SLAM to propose objects in the environment, and incorporate efficient feature encoding techniques to provide an improved object recognition solution whose run-time is nearly-constant to the number of objects identifiable by the system. Through various evaluations, we show that our SLAM-aware monocular recognition solution is competitive to current state-of-the-art in the RGB$\mathrm{D}$ object recognition literature. We believe that robots equipped with such a monocular system will be able to robustly recognize and accordingly act on objects in their environment, in spite of object clutter and recognition ambiguity inherent from certain object viewpoint angles.

\section{ACKNOWLEDGMENTS}

This work was funded by the Office of Naval Research under grants MURI N00014-10-1-0936, N00014-11-1-0688 and N00014-13-1-0588 and by the National Science Foundation under Award IIS-1318392. We would like to thank the authors of ORB-SLAM and LSD-SLAM for providing source code of their work, and the authors of the UWRGB-D Dataset [24, 25] for their considerable efforts in collecting, annotating and developing benchmarks for the dataset. 


\section{REFERENCES}

[1] R. Arandjelovic and A. Zisserman. All about VLAD. In Proc. IEEE Conf. on Computer Vision and Pattern Recognition (CVPR). IEEE, 2013.

[2] S. Y. Bao and S. Savarese. Semantic structure from motion. In Proc. IEEE Conf. on Computer Vision and Pattern Recognition (CVPR). IEEE, 2011.

[3] S. Y. Bao, M. Bagra, Y.-W. Chao, and S. Savarese. Semantic structure from motion with points, regions, and objects. In Proc. IEEE Conf. on Computer Vision and Pattern Recognition (CVPR). IEEE, 2012.

[4] L. Bo, X. Ren, and D. Fox. Hierarchical matching pursuit for image classification: Architecture and fast algorithms. In Advances in Neural Information Processing Systems (NIPS), 2011.

[5] A. Bosch, A. Zisserman, and X. Muoz. Image classification using random forests and ferns. In Proc. Int'l. Conf. on Computer Vision (ICCV). IEEE, 2007.

[6] J. Carreira and C. Sminchisescu. Constrained parametric min-cuts for automatic object segmentation. In Proc. IEEE Conf. on Computer Vision and Pattern Recognition (CVPR). IEEE, 2010.

[7] R. O. Castle, G. Klein, and D. W. Murray. Combining monoSLAM with object recognition for scene augmentation using a wearable camera. Image and Vision Computing, 28(11), 2010.

[8] K. Chatfield, V. Lempitsky, A. Vedaldi, and A. Zisserman. The devil is in the details: an evaluation of recent feature encoding methods. In Proceedings of the British Machine Vision Conference (BMVC), 2011.

[9] M.-M. Cheng, Z. Zhang, W.-Y. Lin, and P. Torr. BING: Binarized normed gradients for objectness estimation at 300fps. In Proc. IEEE Conf. on Computer Vision and Pattern Recognition (CVPR), 2014.

[10] J. Civera, D. Gálvez-López, L. Riazuelo, J. D. Tardós, and J. Montiel. Towards semantic SLAM using a monocular camera. In Proc. IEEE/RSJ Int'l Conf. on Intelligent Robots and Systems (IROS). IEEE, 2011.

[11] A. Collet and S. S. Srinivasa. Efficient multi-view object recognition and full pose estimation. In Proc. IEEE Int'l Conf. on Robotics and Automation (ICRA). IEEE, 2010.

[12] G. Csurka, C. Dance, L. Fan, J. Willamowski, and C. Bray. Visual categorization with bags of keypoints. In Workshop on statistical learning in computer vision, ECCV, volume 1, 2004.

[13] N. Dalal and B. Triggs. Histograms of oriented gradients for human detection. In Proc. IEEE Conf. on Computer Vision and Pattern Recognition (CVPR). IEEE, 2005.

[14] T. Dean, M. A. Ruzon, M. Segal, J. Shlens, S. Vijayanarasimhan, and J. Yagnik. Fast, accurate detection of 100,000 object classes on a single machine. In Proc. IEEE Conf. on Computer Vision and Pattern Recognition (CVPR). IEEE, 2013.

[15] J. Delhumeau, P.-H. Gosselin, H. Jégou, and P. Pérez. Revisiting the VLAD image representation. In Proceedings of the 21st ACM international conference on Multimedia, 2013.

[16] J. Engel, T. Schöps, and D. Cremers. LSD-SLAM: Largescale direct monocular SLAM. In Proc. European Conf. on Computer Vision (ECCV). Springer, 2014.

[17] M. Everingham, L. Van Gool, C. K. Williams, J. Winn, and A. Zisserman. The PASCAL Visual Object Classes (VOC) Challenge. Int'l J. of Computer Vision, 88(2):303-338, 2010.

[18] P. F. Felzenszwalb, R. B. Girshick, D. McAllester, and D. Ramanan. Object detection with discriminatively trained part-based models. IEEE Trans. on Pattern Analysis and Machine Intelligence (PAMI), 2010.
[19] C. Forster, M. Pizzoli, and D. Scaramuzza. SVO: Fast semi-direct monocular visual odometry. In Proc. IEEE Int'l Conf. on Robotics and Automation (ICRA), pages 15-22. IEEE, 2014

[20] S. Gupta, R. Girshick, P. Arbelaez, and J. Malik. Learning rich features from RGB-D images for object detection and segmentation. In Proc. European Conf. on Computer Vision (ECCV). 2014.

[21] J. Hosang, R. Benenson, and B. Schiele. How good are detection proposals, really? In M. Valstar, A. French, and T. Pridmore, editors, Proceedings of the British Machine Vision Conference. BMVA Press, 2014.

[22] H. Jégou, M. Douze, C. Schmid, and P. Pérez. Aggregating local descriptors into a compact image representation. In Proc. IEEE Conf. on Computer Vision and Pattern Recognition (CVPR). IEEE, 2010.

[23] K. Lai, L. Bo, X. Ren, and D. Fox. A large-scale hierarchical multi-view RGB-D object dataset. In Proc. IEEE Int'l Conf. on Robotics and Automation (ICRA). IEEE, 2011.

[24] K. Lai, L. Bo, X. Ren, and D. Fox. Detection-based object labeling in 3D scenes. In Proc. IEEE Int'l Conf. on Robotics and Automation (ICRA). IEEE, 2012.

[25] K. Lai, L. Bo, and D. Fox. Unsupervised feature learning for 3D scene labeling. In Proc. IEEE Int'l Conf. on Robotics and Automation (ICRA). IEEE, 2014.

[26] S. Lazebnik, C. Schmid, and J. Ponce. Beyond bags of features: Spatial pyramid matching for recognizing natural scene categories. In Proc. IEEE Conf. on Computer Vision and Pattern Recognition (CVPR), volume 2. IEEE, 2006.

[27] R. Mur-Artal, J. Montiel, and J. D. Tardos. ORB-SLAM: a versatile and accurate monocular SLAM system. arXiv preprint arXiv:1502.00956, 2015.

[28] F. Perronnin, J. Sánchez, and T. Mensink. Improving the fisher kernel for large-scale image classification. In Proc. European Conf. on Computer Vision (ECCV). Springer, 2010.

[29] O. Russakovsky, J. Deng, H. Su, J. Krause, S. Satheesh, S. Ma, Z. Huang, A. Karpathy, A. Khosla, M. Bernstein, A. C. Berg, and L. Fei-Fei. ImageNet Large Scale Visual Recognition Challenge. International Journal of Computer Vision (IJCV), 2015

[30] R. F. Salas-Moreno, R. A. Newcombe, H. Strasdat, P. H. Kelly, and A. J. Davison. SLAM++: Simultaneous localisation and mapping at the level of objects. In Proc. IEEE Conf. on Computer Vision and Pattern Recognition (CVPR). IEEE, 2013.

[31] J. Sivic and A. Zisserman. Video google: A text retrieval approach to object matching in videos. In Proc. Int'l. Conf. on Computer Vision (ICCV). IEEE, 2003.

[32] A. Thomas, V. Ferrar, B. Leibe, T. Tuytelaars, B. Schiel, and L. Van Gool. Towards multi-view object class detection. In Proc. IEEE Conf. on Computer Vision and Pattern Recognition (CVPR), volume 2. IEEE, 2006.

[33] J. R. Uijlings, K. E. van de Sande, T. Gevers, and A. W. Smeulders. Selective search for object recognition. Int'l J. of Computer Vision, 104(2), 2013.

[34] K. E. van de Sande, C. G. Snoek, and A. W. Smeulders. Fisher and VLAD with FLAIR. In Proc. IEEE Conf. on Computer Vision and Pattern Recognition (CVPR). IEEE, 2014.

[35] X. Zhou, K. Yu, T. Zhang, and T. S. Huang. Image classification using super-vector coding of local image descriptors. In Proc. European Conf. on Computer Vision (ECCV). Springer, 2010.

[36] C. L. Zitnick and P. Dollár. Edge boxes: Locating object proposals from edges. In Proc. European Conf. on Computer Vision (ECCV). Springer, 2014. 